\title{
Direct and Reciprocal Space Properties of the Generalized Penrose Tiling
}

\author{
M. ChOdýn ${ }^{a, *}$, P. KuCZera ${ }^{a, b}$ AND J. WOLnY ${ }^{a}$ \\ ${ }^{a}$ Faculty of Physics and Applied Computer Science, AGH University of Science and Technology \\ Al. A. Mickiewicza 30, 30-059 Krakow, Poland \\ ${ }^{b}$ Laboratory of Crystallography, ETH Zurich, Switzerland
}

\begin{abstract}
We have constructed a generalized Penrose tiling by the cut-and-project method and compared its structure with that of the regular Penrose tiling. We derived the structure factor of the generalized Penrose tiling and applied it to calculate the diffraction pattern of a non-decorated structure.
\end{abstract}

DOI: $10.12693 /$ APhysPolA.126.442

PACS: $61.44 . \mathrm{Br}$

\section{Introduction}

The pentagonal Penrose tiling P1 (PPT in what follows) was introduced by Roger Penrose and published in 1974 [1]. This tiling contains five prototiles. Eventually, Penrose reduced the number of prototiles to two convex ones and thus constructed the P3 tiling, better known as the rhombic Penrose tiling (RPT in what follows) [2]. Both these tilings belong to the Penrose LI class (for "local isomorphism" or much better "local indistiguishability") of tilings (PLI in what follows) along with some other tilings like the hexagon-boat-star (HBS) tiling or the kite and dart tiling P2. It has been shown by Kuczera et al. [3] that for the structure analysis of decagonal quasicrystals (dQCs) it is the easiest to use the decorated RPT; however, but for a better explanation and understanding of the structure the decoration of the prototiles can be "translated" to any other tiling within the PLI class. This means that the long range order is the same for all PLI tilings. Hereafter, whenever the term "Penrose tiling" is used, it refers to the rhombic Penrose tiling.

PT can be used as a model for the refinement procedure of dQCs. It has been successfully applied to the structure optimization of various decagonal phases $[3,4]$. GPT [5] is an extension of PT, its prototiles are still the two Penrose rhombs and the local fivefold symmetry is preserved. However, its matching rules and its structure depend on a certain continuous parameter ranging from 0 to 1 . Therefore, there is an uncountable infinity of different GPTs. GPT is a promising alternative instead of $\mathrm{PT}$ as a quasilattice for the description dQC structures; it would allow to go beyond the PLI class of tilings.

\section{Generalized Penrose tiling}

In the higher dimensional $(n \mathrm{D})$ approach, $\mathrm{PT}$ is obtained by projecting a $5 \mathrm{D}$ hypercubic lattice through a

\footnotetext{
*corresponding author; e-mail: maciej.chodyn@gmail.com
}

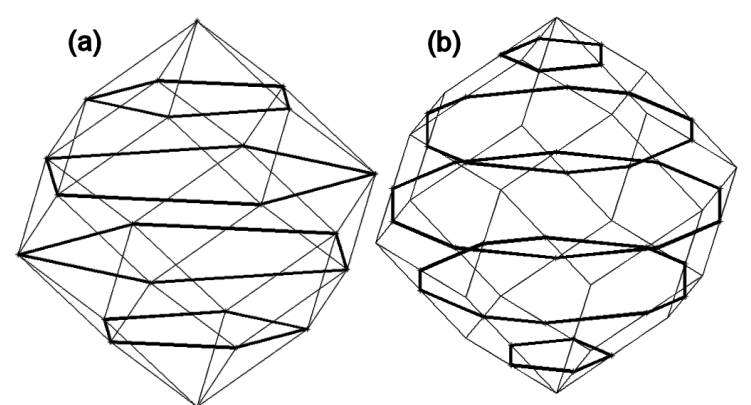

Fig. 1. (a) The ASs of PT, (b) the ASs of GPT, shift $=0.5$.

window consisting of four pentagons, called the atomic surfaces (ASs) in the perpendicular space. The vertices of these pentagons together with two additional points form a rhombicosahedron (Fig. 1a). The shape of the ASs is an effect of positioning the projection strip in $n \mathrm{D}$ space in such a way, that it contains one 5D hypercubic unit cell and its boundaries are in the nodes of the lattice. Shifting the projection strip by a unit vector along the body diagonal of the rhombicosahedron will result in a coordinate change of the vertices of the ASs while their shape will remain unchanged and the structure generated by a projection through such a window will still be the PT.

The GPT is obtained by shifting the projection strip along the body diagonal of the rhombicosahedron by a fraction of a unit vector. The ASs constructed in this way will still be bounded by the same rhombicosahedron. However, their vertices will no longer match the vertices of the rhombicosahedron (Fig. 1b). In other words the ASs in Fig. 1a are shifted along the body diagonal of the rhombicosahedron $[6,7]$. Three of the previously pentagonal ASs will become decagons (equilateral only for the shift equal to 0.5 ), one will remain pentagonal, and additionally one more pentagon will be created (for PT it is a single point). A projection of the $5 \mathrm{D}$ hypercubic lattice through a window consisting of these five polygons will generate the GPT whose structure will depend on the 
shift parameter. Two examples of GPT, for shifts equal to 0.2 and 0.5 are shown in Fig. $2 \mathrm{a}$ and b, respectively.
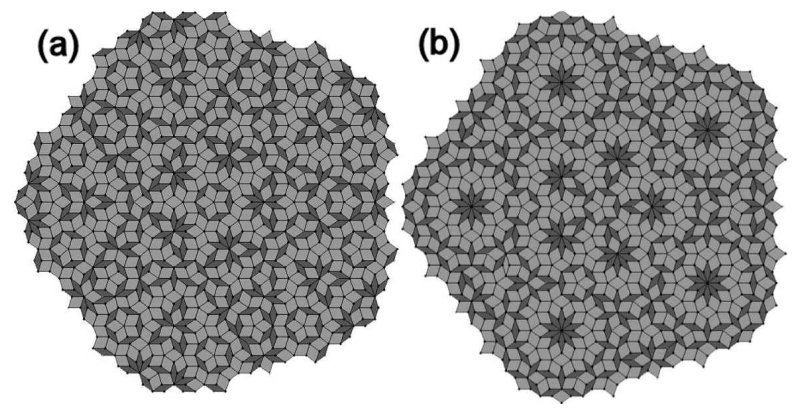

Fig. 2. (a) The GPT, shift $=0.2$, (b) the GPT, shift $=0.5$.

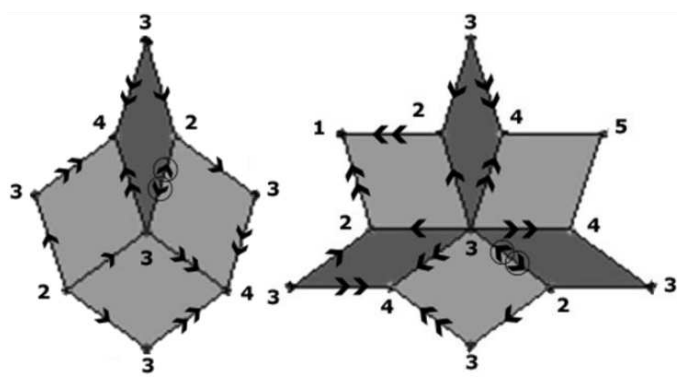

Fig. 3. Examples of PT matching rules breaking in the GPT.

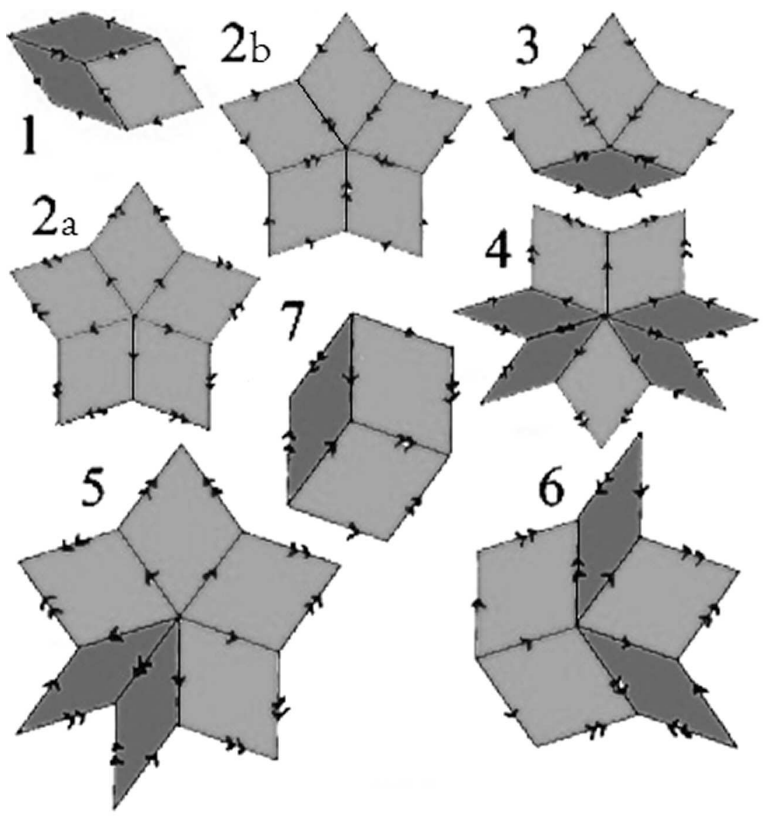

Fig. 4. Rhombs configurations in PT.

The prototiles of the GPT are still thick and thin rhombs but the matching rules of the tiling are different. The PT rules are not applicable to GPT (Fig. 3). The eight possible vertex configurations of PT (Fig. 4) are not sufficient any more, new vertex configurations

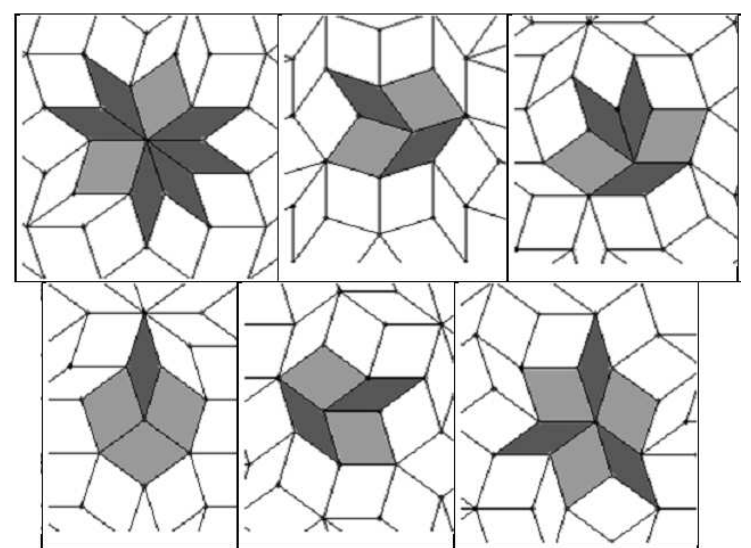

Fig. 5. Examples of new rhombs configurations in GPT, shift $=0.2$.

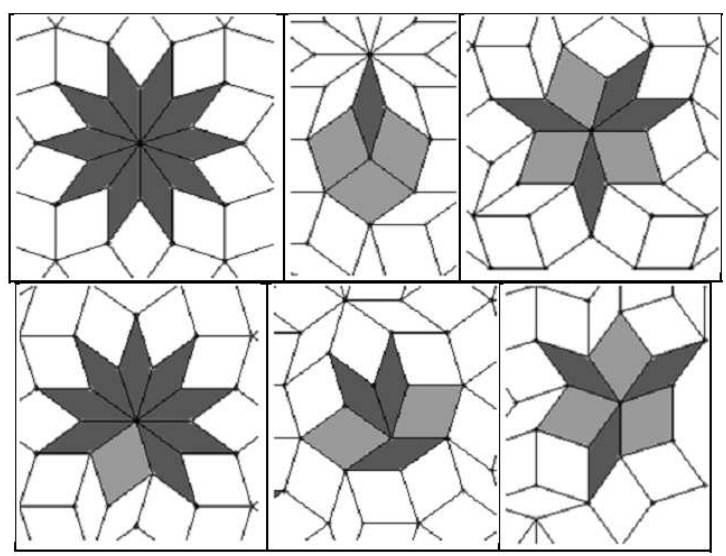

Fig. 6. Examples of new rhombs configurations in GPT, shift $=0.5$.

are the result of the shape change of ASs and the appearance of a new AS (additional pentagon - upper or lower in Fig. 1b). A few examples of new vertex configurations are shown in Figs. 5 and 6 . For each value of the shift parameter different set of vertex configurations (vertex atlas) will be created.

For each of the two presented GPTs we have calculated the average unit cell (AUC) [8] using the reciprocal space vectors of indices $(-1,-3,-3,-1),(2,1,-1,-2)$, and projecting 6000 points. The obtained probability

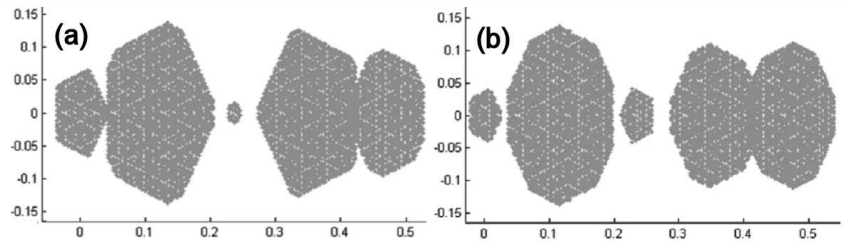

Fig. 7. (a) The AUC for GPT, shift $z=0.2$, (b) the AUC for GPT, shift $z=0.5$. 
distributions (Fig. 7a and b) show the shapes of three decagons and two pentagons that correspond to the shape of ASs. The result was to be expected, because the AUC is an oblique projection of the ASs onto physical space [8].

\section{Structure factor}

The derivation of an analytical formula for structure factor for an empty GPT tiling (with no decoration) is made similarly to the derivation for the PT [9]. The positions of GPT diffraction peaks are identical as in PT and given by [9]:

$$
\begin{aligned}
k_{x} & =\frac{2 \pi}{5 a}\left[-h_{1}-h_{4}+\tau\left(h_{1}-h_{2}-h_{3}+h_{4}\right)\right], \\
k_{y} & =\frac{2 \pi}{5 a} \sqrt{\tau+2}\left[h_{1}-h_{2}+h_{3}-h_{4}+\tau\left(h_{2}-h_{3}\right)\right], \\
k_{x_{\perp}} & =\frac{2 \pi}{5 a}\left[-h_{2}-h_{3}+\tau\left(-h_{1}+h_{2}+h_{3}-h_{4}\right)\right] \\
k_{y_{\perp}} & =\frac{2 \pi}{5 a} \sqrt{\tau+2}\left[-h_{1}-h_{2}+h_{3}+h_{4}+\tau\left(h_{1}-h_{4}\right)\right], \\
k_{z \perp} & =\frac{2 \pi}{5 a}\left(h_{1}+h_{2}+h_{3}+h_{4}\right) .
\end{aligned}
$$

Their intensities are calculated as the square modulus of the structure factor $F$. We start the structure factor calculation from its definition:

$$
F\left(k_{x}, k_{y}\right)=\sum_{j=1}^{\infty} \exp \left(\mathrm{i}\left(k_{x} x_{j}+k_{y} y_{j}\right)\right),
$$

where $\left(k_{x}, k_{y}\right)$ is the reciprocal space vector in physical space $\left(x_{j}, y_{j}\right)$ is the position of the $j$-th atom in physical space.

We can use the following relationship to move from physical space to perpendicular space: $\exp (\mathrm{i} \boldsymbol{k} \cdot \boldsymbol{r})=1$. This allows us to write

$$
k_{x} x+k_{y} y=-\left(k_{x_{\perp}} x_{\perp}+k_{y_{\perp}} y_{\perp}+k_{z \perp} z_{\perp}\right),
$$

where $\left(k_{x_{\perp}}, k_{y_{\perp}}, k_{z \perp}\right)$ is a reciprocal space vector in perpendicular space $\left(x_{\perp}, y_{\perp}, z_{\perp}\right)$ is the position of an atom in perpendicular space.

In spite of the fact that we calculate the structure factor for a reciprocal space vector in physical space the structure factor value now only depends on the perpendicular part of the reciprocal space vector

$$
F\left(k_{x}, k_{y}\right)=\sum_{j=1}^{\infty} \exp \left(-\mathrm{i}\left(k_{x_{\perp}} x_{\perp}+k_{y_{\perp}} y_{\perp}+k_{z \perp} z_{\perp}\right)\right) \text {. }
$$

This infinite sum is confined to a region bounded by the edges of the ASs. Points in this region are distributed quasicontinuously; this allows us to replace the sum by an integral. At the same time $z_{\perp}$ has only four values for PT and five for GPT; this in turn allows us to write it as a sum of five surface integrals. In addition, because we calculate the transform for an empty GPT lattice we can simplify Eq. (4) to

$$
\begin{aligned}
& F\left(k_{x}, k_{y}\right)=C \sum_{z_{\perp}=1}^{5} \exp \left(-\mathrm{i} k_{z \perp} z_{\perp}\right) \\
& \quad \times \iint_{\mathrm{AS}} \exp \left(-\mathrm{i}\left(k_{x_{\perp}} x_{\perp}+k_{y_{\perp}} y_{\perp}\right)\right) \mathrm{d} x_{\perp} \mathrm{d} y_{\perp},
\end{aligned}
$$

$$
k_{z \perp} z_{\perp}=\frac{2 \pi}{5}\left(h_{1}+h_{2}+h_{3}+h_{4}\right) z_{\perp} .
$$

The product of $k_{z \perp} z_{\perp}$ is equal to the multiples of $0.4 \pi$ and can only be equal to one of the five values: $0,0.4 \pi$, $0.8 \pi, 1.2 \pi$ or $1.6 \pi$.

The next step is the calculation of the surface integrals over the ASs. The ASs can be divided into triangles. The transform $T$ of a single triangle is given by

$$
T=\iint_{\Delta} \exp \left(-\mathrm{i}\left(k_{x_{\perp}} x_{\perp}+k_{y_{\perp}} y_{\perp}\right)\right] \mathrm{d} x_{\perp} \mathrm{d} y_{\perp} .
$$

After integration we obtain

$$
\begin{aligned}
T & =\frac{1}{k_{y_{\perp}}}\left[D_{12}\left(E_{2}-E_{1}\right)+D_{23}\left(E_{3}-E_{2}\right)\right. \\
& \left.-D_{13}\left(E_{3}-E_{1}\right)\right],
\end{aligned}
$$

where

$$
\begin{aligned}
E_{j} & =\exp \left(-\mathrm{i}\left(k_{x_{\perp}} x_{j_{\perp}}+k_{y_{\perp}} y_{j_{\perp}}\right)\right), \\
D_{i j} & =\frac{1}{k_{x_{\perp}}+k_{y_{\perp}} a_{i j}}, a_{i j}=\frac{y_{i_{\perp}}-y_{j_{\perp}}}{x_{i_{\perp}}-x_{j_{\perp}}}
\end{aligned}
$$

and $\left(x_{i_{\perp}} y_{i_{\perp}}\right)$ are the positions of ASs vertices in perpendicular space.

The following result may be used to calculate the transform of a pentagon or decagon by substituting the coordinates of the remaining triangles. The formula can be further simplified by noticing that the element of $D_{12}\left(E_{2}-E_{1}\right)$ for triangle $j$ is equal to the $-D_{13}\left(E_{3}-E_{1}\right)$ element of the triangle $j+1$.

The resulting structure factor is given by formula (10). It allows us to exactly calculate its value for a given reciprocal space vector $\left(k_{x}, k_{y}\right)$ without numerical calculation of integrals

$$
\begin{aligned}
& F\left(k_{x}, k_{y}\right)=C \sum_{z_{\perp}=1}^{5}\left[\exp \left(-\mathrm{i} k_{z \perp} z_{\perp}\right)\right. \\
& \left.\quad \times \sum_{j=0}^{N} \frac{D_{j, j+1}\left(E_{j+1}-E_{j}\right)}{k_{y_{\perp}}}\right],
\end{aligned}
$$

where $N$ is equal to 4 or 9 depending on whether $\mathrm{AS}$ is a pentagon or decagon.

\section{Diffraction pattern}

The diffraction pattern obtained from the structure factor is shown in Fig. 8a and compared with the pattern obtained by numerical calculation shown in Fig. 8b. The resulting patterns are consistent. From the comparison between diffraction patterns for GPT and PT with no decoration one can conclude that the positions of diffraction peaks are the same but their intensities are slightly changed: $0-2 \%$ of the peak value for $\boldsymbol{k}=0$. The relative intensity change is higher, mainly for the weaker peaks it can be $20 \%$ or more. Two reflections, $(0,-3,-5,-3)$ and $(3,2,-2,-3)$ were selected to plot of the relative intensity change as a function of the shift (Fig. 9).

Deviations in intensities do not have any numerical reasons, but they are related to the GPT itself. To prove this the structure factor and relative intensity (normalized to the peak at $\boldsymbol{k}=0)$ for peak $(3,2,-2,-3)$ was calculated analytically for PT 


$$
\begin{gathered}
F=\frac{-1}{k_{y_{\perp}}}\left(\frac{10 \tau^{2}}{\pi}-\frac{5 \tau^{2}}{\pi} \cos \alpha+\frac{10 \tau^{4}}{\pi} \cos \tau \alpha\right. \\
\left.+\frac{5 \tau^{5}}{\pi} \cos \frac{\alpha}{\tau}\right) \approx 15.522, I[\%] \approx 81.393,
\end{gathered}
$$

where $\alpha=\frac{2 \pi(\tau+2)}{5 \tau^{4}}$ and $\tau=\frac{1+\sqrt{5}}{2}$ are constants.

On the other hand, for GPT with a shift of 0.5 we obtain

$$
\begin{aligned}
F & =\frac{1}{k_{y_{\perp}}}\left[\frac{10 \tau^{3}}{\pi} \cos \frac{\alpha}{2}-\frac{10 \tau^{4}}{\pi} \cos \frac{\alpha}{2 \tau}+\frac{10 \tau^{4}}{\pi} \cos \frac{\alpha \tau^{2}}{2}\right. \\
& -\frac{5 \tau^{2}}{\pi}-\frac{10 \tau^{3}}{\pi} \cos \alpha-\frac{10 \tau^{3}}{\pi} \cos \frac{5 \tau \alpha}{2(\tau+2)} \\
& \left.+\frac{5 \tau^{5}}{\pi} \cos (\tau \alpha)\right] \approx 15.521, I[\%] \approx 81.380
\end{aligned}
$$

Comparing these two formulae shows that while the two numerical values are similar, the structure factor and the intensities of the unshifted PT and the GPT obey different analytical formulae.

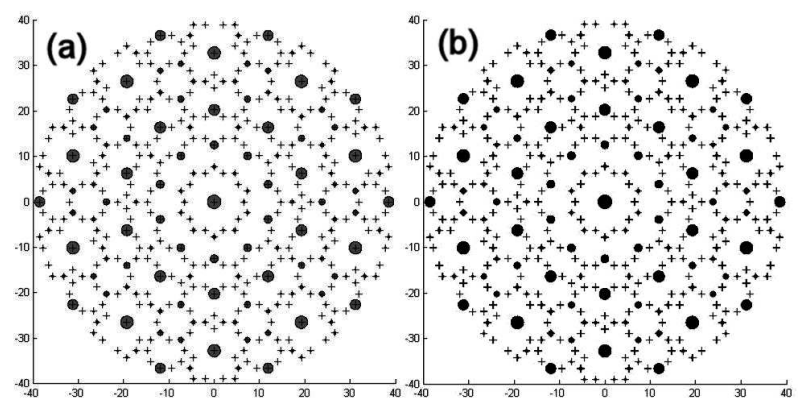

Fig. 8. (a) Diffraction pattern of GPT, shift $=0.5$, calculated from structure factor, (b) diffraction pattern of GPT, shift $=0.5$, numerical calculation

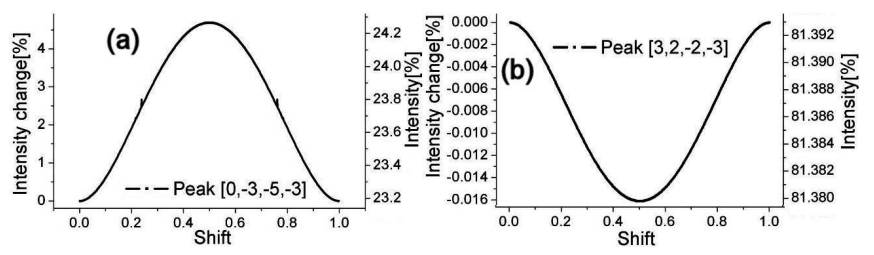

Fig. 9. Relative intensity change as a function of shift.

\section{Conclusions}

The GPT was obtained by projecting the 5D hypercubic lattice through a window consisting of five polygons. The resulting structure is very similar to that of the PT, but its matching rules and vertex atlases are different. The structure factor for GPT has been derived and used for calculation of the diffraction pattern of a non-decorated structure. The peaks of GPT are in identical positions as those of PT, but their intensities are changed as a result of shift of the ASs. The relative change of the weaker peaks is much larger than that of the strong ones. The next step would be to derive the structure factor for arbitrarily decorated GPT using the AUC approach and to try to use the shift of the ASs as one of the parameters in the structure refinement procedure of dQCs. This would allow to optimize also the long-range order and has not been tried before.

\section{Acknowledgments}

We acknowledge partial support by Polish National Science Centre (NCN) under grant No. DEC2013/11/B/ST3/03787.

\section{References}

[1] R. Penrose, Bull. Inst. Math. Appl. 10, 266 (1974).

[2] R. Penrose, Math. Intelligencer 2, 32 (1979).

[3] P. Kuczera, J. Wolny, W. Steurer, Acta Crystallogr. $B$ 68, 578 (2012).

[4] P. Kuczera, J. Wolny, F. Fleischer, W. Steurer, Philos. Mag. 91, 2500 (2011).

[5] M. Kleman, A. Pavlovitch, J. Phys. A: Math. Gen. 20, 687 (1987).

[6] W. Steurer, S. Deloudi, Crystallography of Quasicrystals, Springer-Verlag, Berlin 2009.

[7] K.N. Ishihara, A. Yamamoto, Acta Crystallogr. A 44, 508 (1988).

[8] J. Wolny, Philos. Mag. 77, 395 (1998).

[9] B. Kozakowski, J. Wolny, Acta Crystallogr. A 66, 489 (2010). 